\title{
Analysis of Chopped Partial Discharge Sequence
}

\author{
Marek Florkowski \\ ABB Corporate Research \\ 31-038 Kraków, Poland
}

\author{
Barbara Florkowska, Paweł Zydroń \\ AGH University of Science and Technology \\ 30-059 Kraków, Poland
}

\begin{abstract}
In order to investigate partial discharge mechanism and dynamics, a novel methodology - Chopped Partial Discharges - has been elaborated. Typically, either during laboratory measurements or at on-site field testing, partial discharges are detected by applying a continuous sinusoidal excitation, most regularly $50 / 60 \mathrm{~Hz}$ A chopped partial discharge sequence consists of a number of packets of a base waveform, separated by a variable time delay. The base waveform may be sinusoidal, square-like, trapezoidal or with the different shapes reflecting various switching phenomena and stresses occurring in insulation systems. In this paper, a chopped partial discharge approach is applied in the investigation of the internal mechanism inside voids, especially with respect to void surface conditions and charge decay phenomena. The void embedded in epoxy resin was investigated. In order to properly understand the mechanism of partial discharge in a void, it is necessary to understand the field created by surface charges which remain after previous discharge events. Such deposited charges create an internal field, which interplays with superimposed external electric field in terms of the discharge inception conditions. As a result partial discharge inception occurs at a different phase angle of the external background field relative to the equivalent inception angle for a continuous sequence.
\end{abstract}

Keywords- partial discharge mechanism; charge decay; void surface conductivity

\section{INTRODUCTION}

During the last decades, a large amount of progress has been made in understanding the partial discharge (PD) mechanism and the subsequent development of measurement techniques. Unlike classical methods, which are based on continuous voltage stimuli, in this paper the chopped partial discharge sequence has been investigated with the aim of further explaining the PD mechanism. This novel approach is based on the application of a repeating series of packets to a dielectric insulation system, with each packet being composed of a number of base waveforms followed by the delay time between subsequent packets. Then the whole epoch is repeated sequentially. There are various conditions that influence the PD mechanism, including inception, governed by electric field distribution and electron availability, or extinction, which is influenced by the material and surface conditions of internal inclusions called voids. Such surface conditions also impact so-called memory effects associated with the behavior of subsequent discharges. In this paper, a chopped partial discharge approach is applied especially with respect to void surface conditions and charge decay phenomena.

\section{CHOPPED PD SEQUENCE}

Typically, either during laboratory measurements or at onsite field testing, partial discharges are detected by applying a continuous sinusoidal excitation, most regularly $50 / 60 \mathrm{~Hz}$ or higher or lower frequency according to certain standards.

a)

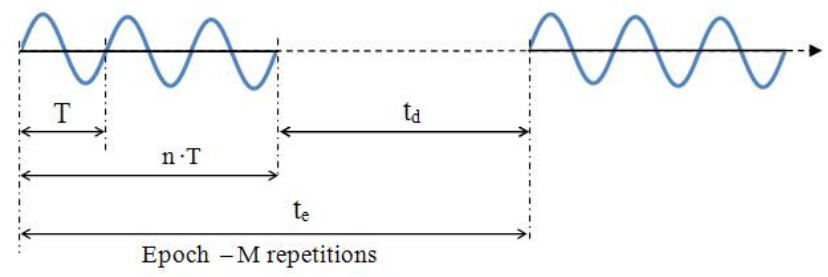

b)

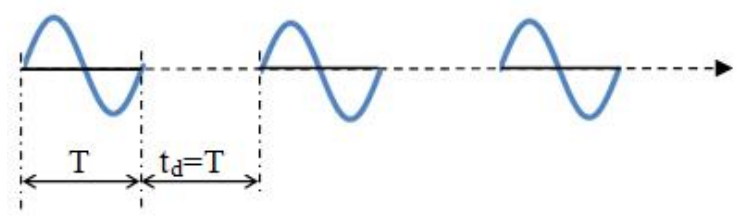

c)

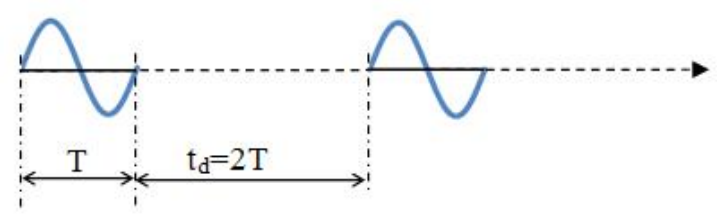

d)

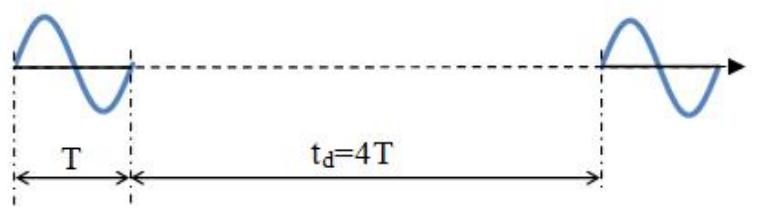

Fig. 1. Chopped partial discharge sequence, a) general methodology, b) 1-period followed by delay time $\left.t_{d}=T, c\right) 1$-period followed by delay time $t_{d}=2 T$, d) 1-period followed by delay time $t_{d}=4 T$; $T$-period of base waveform, $t_{d}$-delay time, $t_{e}$-duration of the epoch.

In a continuous PD sequence the "first" period differs from subsequent periods in terms of the internal voltage division and the internal phase shift between the applied voltage and internal voltage in the void. Thus in the first period without previous history, the PD "comb" in the leading half period is partial when compared to the continuous sequence. After the occurrence of discharge, the electrons deposited on the void walls undergo decay. Depending on the material they may be trapped and/or discharged on the surface $[2,3,4]$. 
Additionally, such deposited charges create an internal field which interacts with superimposed external electric field in terms of the discharge inception conditions. In order to analyze the internal relaxation phenomena occurring in the void, the novel methodology - Chopped PD (CPD) - is proposed. The chopped PD sequence presented in Fig. 1a consists of a packet of $n$ number of base waveforms, having period $T$, separated by the delay time $t_{d}$. The base waveform can be sinusoidal, square-like, trapezoidal etc., depending on the investigated case. It may also reflect fast switching phenomena and stresses occurring in insulation systems exposed to, for example, power electronics [1]. During the delay time there is no voltage on the specimen or test object. The whole epoch, consisting of base periods and delay time, is then repeated $M$ times during a whole measurement time $t_{m}$. The measurement time is thus defined as:

$$
\mathrm{t}_{\mathrm{m}}=\mathrm{M} \cdot\left(\mathrm{n} \cdot \mathrm{T}+\mathrm{t}_{\mathrm{d}}\right)
$$

In a simple form, the sequence can consist of 1-period of the base waveform e.g. sinusoid, followed by the delay time $t_{d}$, as illustrated in Fig. 1b. The delay time may take any value or can be related to the period of base waveform as shown in Fig. 1b-d. The start of the base waveform may be either normal or inversed, i.e starting from negative halfwave. In a simple CPD sequence the sinusoidal and delay periods can be interleaved and described as the fill factor, for example for $\mathrm{T}=20 \mathrm{~ms}$ and delay time $\mathrm{t}_{\mathrm{d}}=20 \mathrm{~ms}$, the fill factor will be $1: 2$. Similarly if one takes $t_{d}=7 T=140 \mathrm{~ms}$, the fill factor will be $1: 8$, etc. The measurements can be performed in a mode when the measurement time is kept constant while changing the fill factors or one can assume a constant exposure to stress in terms of voltage periods, then the measurement time will be respectively longer with increased delay time. In a chopped PD sequence, assuming the repetition of a packet containing one base waveform, the corresponding PD pattern reflects the acquisition of only so-called "first" periods in the case of a continuous sequence. This means that there is no internal shift between the first and consecutive half waves.

\section{SPECIMENS AND MEASUREMENT SETUP}

Two types of specimens were prepared for laboratory investigations, one having a semiconductive surface on both sides of the void and the second specimen including voids embedded in epoxy resin. The semiconductive void was formed by a layer of insulation paper $(0.12 \mathrm{~mm})$ with a circular void (radius $17.5 \mathrm{~mm}$ ) clamped between two glass plates $2 \mathrm{~mm}$ thick each. The glass plates were painted with semiconductive glue. The epoxy resin embedded void had a radius of $17.5 \mathrm{~mm}$ and a thickness $0.1 \mathrm{~mm}$, while the epoxy sample thickness was $1.1 \mathrm{~mm}$. The electrode arrangements consisted of plane stainless steel electrodes $40 \mathrm{~mm}$ in diameter. During the measurement process, both samples were placed in an oil tank. The PD measurements were performed at $50 \mathrm{~Hz}$ in a phase-resolved mode, with $\mathrm{D}(\varphi, q, n)$ pattern acquisition. PD pulses were synchronized with zero phase angle of testing voltage and accumulated in $256 \times 256$ matrix during a measurement time $t_{m}=60$ second. The chopped sequence was programmed in the waveform generator, which controlled the high voltage amplifier (TREK 20/20B). The detected PD signal from coupling impedance was entered to the signal conditioning unit containing filters and a preamplifier. The synchronization signal for PD acquisition was obtained from a resistive divider.

\section{RESULTS AND OBSERVATIONS}

The electric field inside the void $E_{v}$ is a superposition of applied external field $E_{0}$ (corresponding to the test voltage), transposed according to the field distribution and the shape of the void by the factor $f$ and the internal field $E_{q}$ created by the charges deposited on the void surface during a previous event:

$$
E_{v}=f E_{o}+E_{q}\left(\tau_{q}\right)
$$

where $\tau_{q}$ represents the cumulative decay time constant of the $E_{q}$ field in the void.

The measurements were executed in two modes, continuous sinusoidal sequence and chopped one. The chopped PD measurements were based on a sinusoidal base waveform, in a sequence presented in Fig.1, containing packet of $n=1$ base sinusoidal waveforms, having period $\mathrm{T}=20 \mathrm{~ms}$, separated by the delay time $t_{d}$ being a multiple of $\mathrm{T}$. The duration of a single epoch is given by:

$$
\mathrm{t}_{\mathrm{e}}=\mathrm{T}+\mathrm{kT}=\mathrm{T}(1+\mathrm{k})
$$

where $k$ is the delay multiple. The following nomenclature for a sequence fill factor $f f$ will be assumed:

$$
\mathrm{ff}=\mathrm{nT} / \mathrm{t}_{\mathrm{e}}=\mathrm{n} /(1+\mathrm{k})
$$

thus for $\mathrm{n}=1$ and $\mathrm{k}=1 \mathrm{ff}=1: 2 ; \mathrm{k}=3 \mathrm{ff}=1: 4 ; \mathrm{k}=7 \mathrm{ff}=1: 8$, etc. For $\mathrm{k}=0, \mathrm{ff}=1: 1$ represents a continuous sequence (CONT).

The PD inception voltage for void with semiconductive surfaces was at $U_{0}=5.4 \mathrm{kV}_{\text {peak. }}$. The measurements were performed at $1.4 \cdot \mathrm{U}_{\mathrm{o}}$ equal to $7.5 \mathrm{kV}_{\text {peak. }}$. The $\mathrm{PD}$ pattern obtained at continuous sinusoidal voltage (time delay in CPD sequence $t_{d}=0$ ) is shown in Fig. 2a, while introducing chopped PD sequence with $t_{d}=20$ ms, in Fig. $2 b$.

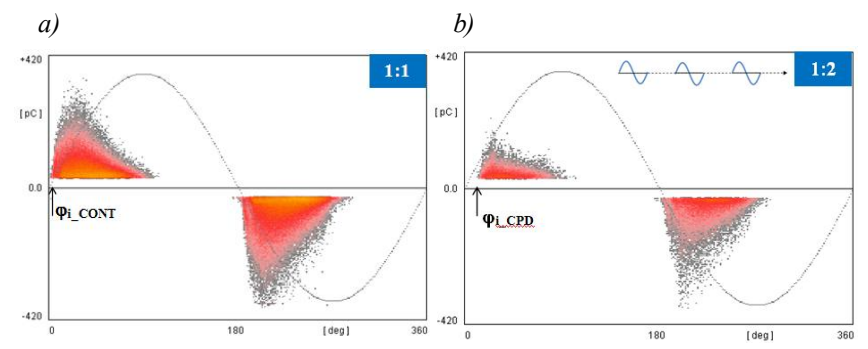

Fig. 2. PD pattern obtained at $7.5 \mathrm{kV}$ in void with semiconductive surfaces: a) continuous sinusoidal voltage $\left(t_{d}=0 \mathrm{~ms}\right), \mathrm{ff}=1: 1, \varphi_{\mathrm{i}_{-} \mathrm{CONT}}=0^{\circ}$,

b) chopped PD pattern $\left(\mathrm{t}_{\mathrm{d}}=20 \mathrm{~ms}\right), \mathrm{ff}=1: 2, \varphi_{\mathrm{i}_{-} \mathrm{CPD}}=14^{\circ}$.

One can see that the negative PD group starts at the same phase angle for both the continuous and chopped sequence, whereas the positive group is also shifted. Thus in the case of delay time $t_{d}=20 \mathrm{~ms}$ between the base packets of test voltage, a phase shift $\Delta \varphi_{i}$ between the inception voltage of the first impulse in the chopped sequence $\left(\varphi_{i_{C} C P D}\right)$ and the inception angle $\left(\varphi_{i}\right.$ CONT $)$ in case of the continuous sequence may be observed.

$$
\Delta \varphi_{\mathrm{i}}=\varphi_{\mathrm{i}} \mathrm{CPD}-\varphi_{\mathrm{i} C \mathrm{CONT}}
$$

The PD inception voltage for void in epoxy resin was at $\mathrm{U}_{\mathrm{o}}=3.0 \mathrm{kV}_{\text {peak }}$. The measurements were performed at $1.4 \cdot \mathrm{U}_{\mathrm{o}}$ 
equal to $4.2 \mathrm{kV}_{\text {peak. }}$ The $\mathrm{PD}$ pattern obtained at continuous sinusoidal voltage is shown in Fig. 3a. Applying CPD sequence with delay time up to $1260 \mathrm{~ms}$, led to the patterns shown in Fig. 3b-d being obtained. One can notice that the positive PD group has changed totally in the chopped sequence relative to the continuous one, resulting in the shift of the whole positive PD group and the radical increase of the maximum charge.

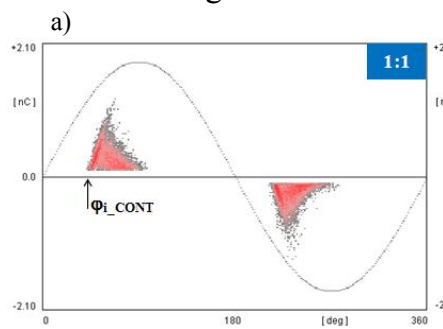

\begin{tabular}{l|l|} 
c) \\
\hline \\
\hline
\end{tabular}

b)

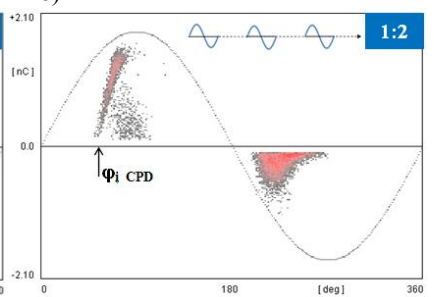

d)
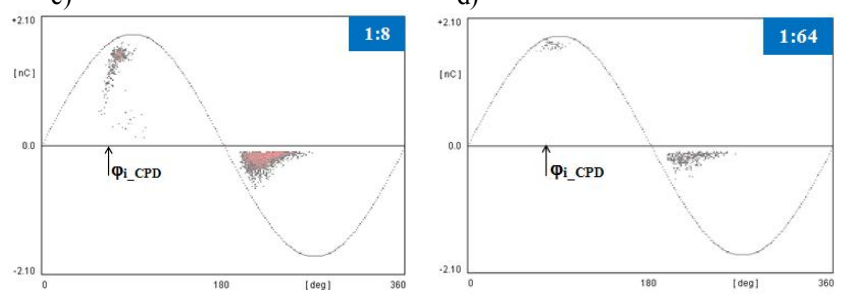

Fig. 3. PD pattern obtained at a) continuous sinusoidal voltage $\left(t_{d}=0 \mathrm{~ms}\right)$ at 4.2 $\mathrm{kV}$ in an epoxy resin void, and chopped PD sequence b) 1:2, c) 1:8, d) 1:64 $\left(\mathrm{t}_{\mathrm{d}}=1260 \mathrm{~ms}\right)$.

In the presented sequence $1: 2,1: 4,1: 8$, up to $1: 64\left(t_{d}=1260\right.$ $\mathrm{ms})$, the inception phase angle is shifting, starting at $\varphi_{\mathrm{CONT}}=41^{\circ}$ for continuous run $(1: 1)$, up to $\varphi_{\mathrm{CPD}}=69^{\circ}$ for chopping 1:64. This means that the total phase shift $\Delta \varphi_{i}$ equals to $28^{\circ}$. For longer time delays than $t_{d}>1260 \mathrm{~ms}$ no further phase shift of the positive PD group was observed. The inception phase angle $\varphi_{i}$ and maximum charge $Q_{\max }{ }^{+}$in the positive half wave of the CPD sequence up to fill factor $f f=1: 64$ for epoxy resin void is shown in Table 1.

TABLE I. INCEPTION PHASE ANGLE AND MAXIMUM CHARGE IN CPD SEQUENCE FOR EPOXY RESIN VOID

\begin{tabular}{|c|c|c|c|c|c|c|c|}
\hline Sequence & $1: 1$ & $1: 2$ & $1: 4$ & $1: 8$ & $1: 16$ & $1: 32$ & $1: 64$ \\
\hline$\left.\varphi_{i}^{+}{ }^{\circ}\right]$ & 41 & 49 & 51 & 59 & 62 & 69 & 69 \\
\hline $\mathrm{Q}_{\max }{ }^{+}[\mathrm{nC}]$ & 1.10 & 1.48 & 1.58 & 1.65 & 1.69 & 1.71 & 1.74 \\
\hline
\end{tabular}

The inception phase angle shift is also indicative of a higher inception voltage, which provides a higher maximum charge in positive cycle. The $Q_{\max }{ }^{+}$in a continuous sequence was equal to $1.1 \mathrm{nC}$ and yields $1.74 \mathrm{nC}$ in chopped one at $\mathrm{ff}=1: 64$. The number of discharges in a continuous versus chopped sequence for an epoxy void is shown in Fig. 4 for measurement time $t_{m}=60$ seconds. The number of $P D$ pulses in the negative half wave $\mathrm{N}^{-}$for chopped sequences is higher when compared with continuous ones up to the $\mathrm{ff}=1: 64$. However, the number of PD in the positive half wave $N^{+}$ drops for CPD when compared to the continuous sequence.

\section{DISCUSSION}

In this paper the CPD sequence was applied to investigate the internal charge decay process in voids having radically different surface conductivities i.e. semiconductive and epoxy.
The first period in the PD acquisition is always different and the CPD methods can extract it from a continuous train of pulses. A key element in the proper understanding of the PD mechanism is the analysis of the field $E_{q}$ created by surface charges which have remained from previous discharge events.

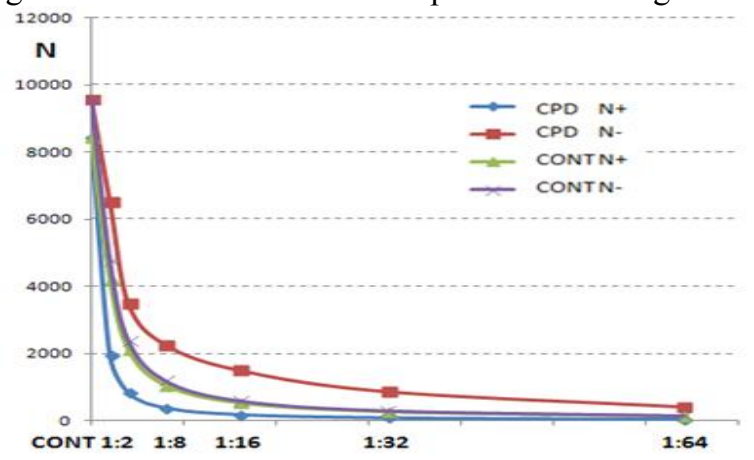

Fig. 4. Number of discharges in continuous versus chopped sequence for epoxy void $\left(\mathrm{t}_{\mathrm{m}}=60 \mathrm{sec}\right)$.

Charge accumulates on the cavity wall after each discharge and, taking into account polarity, impacts the total charge creating $E_{q}$ field. By introducing a time delay after the first period we may in some way "freeze" the internal field $E_{q}$ in the void, coming from the deposited charges at the last discharge and observe its decay, as illustrated at point $\mathrm{A}$ in Fig. 5. Changing the delay time $t_{d}$ between subsequent periods in the CPD sequence, can reveal the process of $E_{q}$ decay. Charge decay and corresponding memory effects in the void have a different origin. One component is related to the surface conductivity which may vary over a broad range [4-7]. The surface charge field $E_{q}$ is directly proportional to the charge deployed by the discharge on a void surface $E_{q} \sim q$ and can be expressed as a decaying function of time:

$$
E_{q}(t)=E_{q 0} \cdot \exp \left(-t / \tau_{q}\right)
$$

where: $E_{q 0}$ is the initial charge value, $\tau_{q}$ represents the cumulative charge $q$ decay due to surface recombination $\tau_{s}$, deeper trapping and bulk conduction $\tau_{t}$ and other drifts $\tau_{d}$. In a continuous PD sequence field $E_{q}$ is involved in the buildup of the electric void field in superposition with the background $f E_{0}$ component. In a chopped PD sequence we are extending the delay time $t_{d}$ and looking for the disappearance of the $E_{q}$ field component. The lack of $E_{q}$ results in a higher inception voltage, as the $E_{q}$ component just after voltage polarity reversal is in phase with $f E_{0}$ field. As a result, there is an inception phase shift $\varphi_{i} C P D$ relative to the inception angle at continuous sequence $\varphi_{i}$ CONT. It is important to note that the elevated inception voltage occurs only for positive half period (or negative in case of inverted CPD sequence). This voltage increase $\Delta U_{i}$ is proportional to the maximum discharge charge as seen in Fig. 3b-d. The number of discharges $N^{+}$in CPD in positive half period drops relative to the continuous one, for all fill factors up to $1: 64$. The increase of inception voltage $\Delta U_{i}$ can be calculated from the phase shift $\Delta \varphi_{\mathrm{i}}=14^{\circ}$ $\left(\varphi_{\mathrm{i} C \mathrm{CONT}}=0^{\circ}, \varphi_{\mathrm{i} \mathrm{CPD}}=14^{\circ}\right)$, as is observed from Fig. 2 .

$$
\Delta U_{\mathrm{i}}=\mathrm{U}_{\mathrm{P}_{-} \text {CONT }}\left[\sin \left(\varphi_{\mathrm{i}} \mathrm{CPD}\right)-\sin \left(\varphi_{\mathrm{i}} \text { CONT }\right)\right]
$$

The peak value of $U_{p_{-} \text {CONT }}=7.5 \mathrm{kV}$, thus the increase of PD inception voltage was $\Delta \mathrm{U}_{\mathrm{i}}=1.8 \mathrm{kV}$. The same effect was 
observed also for inverse sequence, i.e. the sequence starting with negative half wave.

The decay of the $E_{q}\left(\tau_{q}\right)$ field in the void in the Chopped PD sequence is illustrated in Fig. 5 in a context of background field $f E_{0}$ and void field $E_{v}$. The process for high and low surface conductivity is marked.

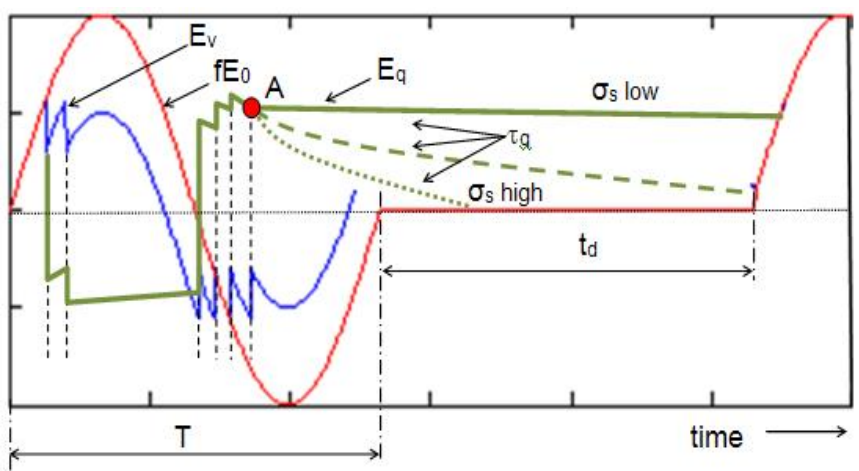

Fig. 5. Decay of $E_{q}$ field in the void in Chopped PD sequence.

At point $\mathrm{A}$ - last discharge in the sequence before delay time $t_{d}$ - the $E_{q}$ field starts to decay. $\mathrm{E}_{\mathrm{v}}$ - field in void, $\mathrm{fE}_{0}-$ background field. Process for high and low surface conductivity is marked.

In the case of the void embedded in the epoxy resin, after applying the chopped sequence one can recognize a radical change of the PD patterns in the positive half period as shown in Fig. 3. The epoxy void surface has relatively high resistivity relative to the semiconductive case, thus the $E_{q}$ related charge decay persists longer. The delay time $t_{d}$ was extended up to the fill factor $\mathrm{ff}=1: 64, \mathrm{t}_{\mathrm{d}}=1260 \mathrm{~ms}$ in order to ascertain the disappearance of the $E_{q}$ field. The corresponding inception phase shift $\varphi_{i}$ is depicted in Fig. 3. The vanishing $E_{q}$ field needs compensation in the form of an elevated inception voltage, resulting in a higher $f E_{0}$ field in the void. The higher inception voltage leads to a lower number of PD pulses in the positive period - trace CPD $N^{+}$in Fig.4. The increase of inception voltage $\Delta U_{i}$ can be calculated from the phase shift $\Delta \varphi_{\mathrm{i}}=28^{\circ}\left(\varphi_{\mathrm{i} C O N T}=41^{\circ}, \varphi_{\mathrm{i}} \mathrm{CPD}=69^{\circ}\right.$ at $\left.1: 64\right)$, as is seen from Fig. 3. The peak value of $U_{p \text { CONT }}=4.2 \mathrm{kV}$, thus the increase of PD inception voltage according to equation (7) is $\Delta \mathrm{U}_{\mathrm{i}}=1.2 \mathrm{kV}$. Due to the higher PD inception voltage in the chopped sequence, the maximum charge increased from $0.9 \mathrm{nC}$ for the continuous case up to $1.7 \mathrm{nC}$ for $f f=1: 64$ in CPD.

The obtained results confirm the relationship between the impact of the chopped PD sequence and the dynamics of charge decay. Under certain conditions, one assumes the dominant influence of surface recombination and derives the estimation of the surface conductivity of the void. Thus, if during the delay time $\mathrm{t}_{\mathrm{d}}=1260 \mathrm{~ms}$ the $E_{q}$ field vanished, one can determine from its decay time constant $\tau_{s}$ the void surface conductivity using equation (8) and supposing that to reach steady state $4 \tau_{s}$ are needed:

$$
\sigma_{\mathrm{s}}=2 \cdot \varepsilon_{0} \cdot \mathrm{r} / \mathrm{t}_{\mathrm{d}}
$$

The epoxy void has a radius $\mathrm{r}=17,5 \mathrm{~mm}$, thus the estimated void wall surface conductivity will be approximately $\sigma_{\mathrm{s}}=2.4 \cdot 10^{-13} \mathrm{~S}$. The void parameters, inception voltage and related phase angle, obtained for semiconductive and epoxy resin voids are shown in Table II.

\begin{tabular}{|c|c|c|c|}
\multicolumn{4}{|c|}{ TABLE II. } \\
\begin{tabular}{|c|c|c|c|}
\hline Void & $\boldsymbol{\sigma}_{\mathrm{s}}[\mathrm{S}]$ & $\Delta \mathrm{U}_{\mathrm{i}}[\mathrm{kV}]$ & $\Delta \boldsymbol{\varphi}_{\mathrm{i}}\left[{ }^{\circ}\right]$ \\
\hline Semiconductive & & 1.8 & 14 \\
\hline Epoxy & $2.4 \cdot 10^{-13}$ & 1.2 & 28 \\
\hline
\end{tabular}
\end{tabular}

In the general case the chopped PD method highlights the internal void condition. The cumulative decay time constant $\tau_{q}$ of $E_{q}$ field may have different components, as was mentioned earlier, which will dominate depending on the material structure. Under certain conditions, the long gap between consecutive voltage periods can lead to the lack of starting electrons and result in time lag and overvoltage. Apart from attempts to estimate absolute surface conductivity, there is a possibility to follow a trend using chopped PD sequence, revealing the deterioration of the void internal structure. Such information might be obtained by comparing a set of CPD patterns recorded in time, revealing the dynamic process occurring during aging and the deterioration of electric insulation systems.

\section{CONCLUSIONS}

This paper presents the application of the novel chopped PD approach to investigations of the internal mechanism inside the voids, especially related to void surface conditions and the charge decay phenomena.

The decay of an internal electric field of deposited charges in a void was revealed and quantified for inclusions with low and high surface conductivities. It can be also concluded that PD inception occurs in chopped sequence at a different phase angle of the external background field relative to the equivalent inception angle for a continuous sequence.

The CPD method creates the potential for various investigations of PD mechanism, behavior and dynamics in high voltage insulating systems. The presented methodology requires further research, however it seems it might have also potential to be even a diagnostic method in the future.

\section{REFERENCES}

[1] M.Florkowski, B.Florkowska, J.Roehrich, P.Zydron, Measurement and analysis of surface partial discharges at semi-square voltage waveforms, IEEE Trans. on Dielect. Electri. Insul., Vol. 18, No 4, pp. 990-996, 2011.

[2] L.A.Dissado, J.C.Fothergill, "Electrical Degradation and Breakdown in Polymers", IET The Institution of Engineering and Technology, 2008.

[3] Y.Shibuya, S.Zoledziowski, J.H.Calderwood, "Void formation and electrical breakdown in epoxy resin", IEEE Trans. on Power Apparatus and Systems, Vol. PAS-96, No. 1, pp. 198-207, 1977.

[4] L.Niemeyer, "A generalized approach to partial discharge modeling", IEEE Trans. Dielectr. Electr. Insul., Vol. 2, Issue 4, pp. 510- 528, 1995.

[5] K.Wu, T.Ijichi, T.Kato, Y.Suzuoki, F.Komori, T.Okamoto, "Contribution of surface conductivity to the current forms of partial discharges in voids", IEEE Trans. Dielectr. Electri. Insul., Vol. 12, Issue 6, pp. 1116 $1124,2005$.

[6] S.Kumara, Y.V.Serdyuk, S.M.Gubanski, "Surface charge decay on polymeric materials under different neutralization modes in air", IEEE Trans. Dielectr. Electr. Insul., Vol. 18, Issue 5, pp.1779-1788, 2011.

[7] I.W. McAllister, "Decay of charge deposited on the wall of gaseous void”, IEEE Trans. Electr. Insul., Vol. 27, Issue 6, pp.1202-1207, 1992. 